\title{
Generalized Derivations Acting as Homomorphisms and Anti- Homomorphisms of Gamma Rings
}

\author{
K. K. Dey" and A. C. Paul \\ Department of Mathematics, Rajshahi University, Rajshahi-6205, Bangladesh
}

Received 2 July 2011, accepted in revised form 1 October 2011

\begin{abstract}
Let $M$ be a prime $\Gamma$-ring and let $I$ be a nonzero ideal of $M$. Suppose that $D: M \rightarrow M$ is a nonzero generalized derivation with associated derivation $d: M \rightarrow M$. Then we prove the following:

(i) If $D$ acts as a homomorphism on $I$, then either $d=0$ on $M$ or $M$ is commutative.

(ii) If $M$ satisfies the assumption (*) (see below), and if $D$ acts as an anti-homomorphism on $I$, then either $d=0$ on $M$ or $M$ is commutative.

Keywords: Prime $\Gamma$-rings; Generalized derivations; Torsion free $\Gamma$-rings; Homomorphisms; Anti-homomorphisms.

(C) 2012 JSR Publications. ISSN: 2070-0237 (Print); 2070-0245 (Online). All rights reserved. doi:10.3329/jsr.v4i1.7911 J. Sci. Res. 4 (1), 33-37 (2012)
\end{abstract}

\section{Introduction}

In classical ring theory, Bell and Kappe [1] proved that if $d$ is a derivation of a semiprime ring $R$ which is either an endomorphism or an anti-endomorphism on $R$, then $d=0$ : whereas, the behavior of $d$ is somewhat restricted in case of prime rings in the way that if $d$ is a derivation of a prime ring $R$ acting as a homomorphism or anti-homomorphism on a nonzero right ideal of $R$, then $d=0$ on $R$.

Afterwards Yenigul and Argac [2] generalized these results with $\alpha$-derivations and $M$. Ashraf, Rehman and Quadri [3] obtained the similar results with $(\sigma, \tau)$-derivations. Analogously Rehman [4] extended the results for generalized derivation acting on nonzero ideals in case of prime rings. Recently Ali and Kumar [5] established the above mentioned result for generalized $(\theta, \varphi)$-derivations in prime rings. By the same motivation, we extend the results in [4] of classical ring theory to the $\Gamma$-ring theory in the case of generalized derivation acts as a homomorphism and an anti-homomorphism of prime $\Gamma$-rings.

\footnotetext{
*Corresponding author: kkdmath@yahoo.com
} 


\section{Definition 1.1 [6]}

Let $M$ and $\Gamma$ be additive abelian groups. $M$ is called a $\Gamma$-ring if for all $x, y, z \in M, \alpha, \beta \in \Gamma$ the following conditions are satisfied:

(i) $x \beta y \in M$,

(ii) $(x+y) \alpha z=x \alpha z+y \alpha z, x(\alpha+\beta) y=x \alpha y+x \beta y, x \alpha(y+z)=x \alpha y+x \alpha z$,

(iii) $(x \alpha y) \beta z=x \alpha(y \beta z)$.

\section{Definition 1.2}

A $\Gamma$-ring $M$ is called prime if for any $a, b \in M, a \Gamma M \Gamma b=0$ implies that either $a=0$ or $b=$ 0 .

\section{Definition 1.3}

An additive mapping $d: M \rightarrow M$ is called a derivation if $d(x \alpha y)=d(x) \alpha y+x \alpha d(y)$ holds for all $x, y \in M, \alpha \in \Gamma$.

For a fixed $a \in M, \alpha \in \Gamma$, the mapping $I_{a}^{\alpha}: M \rightarrow M$ given by $I_{a}^{\alpha}(x)=[x, a]_{\alpha}$ is a derivation which is said to be inner derivation. An additive function $D_{a, b}^{\alpha}: M \rightarrow M$ is called generalized inner derivation if $D^{\alpha}{ }_{a, b}(x)=a \alpha x+x \alpha b$ for some fixed $a, b \in M, \alpha \in \Gamma$. It is straight forward to note that if $D_{a, b}^{\alpha}$ is a generalized inner derivation, then for any $x, y \in M, \alpha \in \Gamma, D_{a, b}^{\alpha}(x \alpha y)=D_{a, b}^{\alpha}(x) \alpha y+x \alpha I_{b}^{\alpha}(y)$ where $I_{b}^{\alpha}$ is an inner derivation. In view of the above observation, the concept of generalized derivation is introduced as follows:

\section{Definition 1.4}

An additive mapping $D: M \rightarrow M$ is called a generalized derivation associated with a derivation d if $D(x \alpha y)=D(x) \alpha y+x \alpha d(y)$ for all $x, y \in M, \alpha \in \Gamma$.

\section{Definition 1.5}

The commutator $x \alpha y-y \alpha x$ will be denoted by $[x, y]_{\alpha}$. We know that $[x \beta y, z]_{\alpha}=[x, z]_{\alpha} \beta y$ $+x \beta[y, z]_{\alpha}+x[\beta, \alpha]_{z} y$ and $[x, y \beta z]_{\alpha}=y \beta[x, z]_{\alpha}+[x, y]_{\alpha} \beta z+y[\beta, \alpha]_{x} z$.

We take an assumption (*) $x \beta z \alpha y=x \alpha z \beta y$ for all $x, y, z \in M$ and $\alpha, \beta \in \Gamma$. Using the assumption the basic commutator identities reduces to $[x \beta y, z]_{\alpha}=[x, z]_{\alpha} \beta y+x \beta[y, z]_{\alpha}$ and $[x, y \beta z]_{\alpha}=y \beta[x, z]_{\alpha}+[x, y]_{\alpha} \beta z$.

\section{Definition 1.6}

Let $M$ be a $\Gamma$-ring. An additive mapping $\phi$ on $M$ is called a homomorphism if $\phi(x, y)=$ $\phi(x) \alpha \phi(y)$, for every $x, y \in M$ and $\alpha \in \Gamma$.

\section{Definition 1.7}

A $\Gamma$-ring $M$ is commutative if $x \alpha y=y \alpha x$ for all $x, y \in M$ and $\alpha \in \Gamma$.

It is clear that a $\Gamma$-ring $M$ is commutative if and only if $[x, y]_{\alpha}=0$ for every $x, y \in M$ and $\alpha \in \Gamma$. 


\section{Definition 1.8}

Let $S$ be a nonempty subset of $M$ and $D$ be a generalized derivation on $M$ with associated derivation $d$. A generalized derivation $D$ of $M$ is said to act as a homomorphism on $S$ if $D(x \alpha y)=D(x) \alpha y+x \alpha d(y)=D(x) \alpha D(y)$ for all $x, y \in S$ and $\alpha \in \Gamma$.

\section{Definition 1.9}

Let $S$ be a nonempty subset of $M$ and $D$ be a generalized derivation on $M$ with associated derivation $d$. A generalized derivation $D$ of $M$ is said to act as an anti-homomorphism on $S$ if $D(x \alpha y)=D(x) \alpha y+x \alpha d(y)=D(y) \alpha D(x)$ for all $x, y \in S$ and $\alpha \in \Gamma$.

\section{Results}

\section{Lemma 2.1}

If $d$ is a nonzero derivation of a prime $\Gamma$-ring $M$, then the left and right annihilators of $d(x)$ $=0, x \in M$. In particular $a \beta[b, x]_{\alpha}=0$ or $[b, x]_{\alpha} \beta a=0$ implies that $I_{b}(x)=0$, $(b \in Z, \alpha, \beta \in \Gamma)$ or $a=0$.

\section{Proof}

In $\operatorname{a\alpha d}(x)=0$ for all $x \in M, \alpha \in \Gamma$, replace $x$ by $x \beta y$. Then we get $\operatorname{a\alpha d}(x \beta y)=0=\operatorname{a\alpha d}(x) \beta y+$ $\operatorname{a\alpha x} \beta d(y)=\operatorname{a\alpha x} \beta d(y)$, for all $x, y \in M, \alpha, \beta \in \Gamma$. If $d \neq 0$, that is $d(y) \neq 0$ for some $y \in M$, then, by the primeness of $M$ we get $a=0$.

\section{Lemma 2.2}

Let $I$ be a nonzero right ideal in a prime $\Gamma$-ring $M$.

(a) If $M$ has a derivation $d$ which is zero on $I$, then $d$ is zero on $M$.

(b) If $M$ has homomorphism $T$ which is the identity on $I$, then $T$ is the identity on $M$.

\section{Proof}

(a) If $d(x)=0, x \in I$, then $0=d(x \alpha r)=d(x) \alpha r+x \alpha d(r)=x \alpha d(r)$, for all $x \in I, r \in M$, $\alpha \in \Gamma$. By lemma 2.1, $d$ must be zero since $I$ is nonzero.

(b) Let $x \in I$ and $a, b \in M$. Then $x \alpha a \beta b=T(x \alpha a \beta b)=T(x \alpha a) \beta T(b)=x \alpha a \beta T(b)$. Thus $x \alpha a \beta(b-T(b))=0$ and either $x=0$ or $b-T(b)=0$. But $I$ is nonzero and so contains an $x \neq 0$. This forces $T(b)=b$ for all $b \in M$.

Lemma 2.3 If a prime $\Gamma$-ring $M$ contains a nonzero commutative right ideal $I$, then $M$ is commutative.

\section{Proof}

If $x \in I$, then $I_{x}(y)=[x, y]_{\alpha}=0$, for all $y \in I, \alpha \in \Gamma$, since $I$ is commutative. By lemma 2.2(a), $I_{x}(y)=0$ on $M$ and $x$ is in the center. Thus $[x, r]_{\alpha}=0$ for every $x \in I, r \in M, \alpha \in \Gamma$. Hence $I_{a}(x)$ $=0$ for $a \in M$ and again by lemma 2.2(a), $I_{a}(r)=0$ and $a$ is in the center for all $a \in M$. Therefore $M$ is commutative. 
Theorem 2.4. Let $M$ be a prime $\Gamma$-ring and $I$ be a nonzero ideal of $M$. Suppose $D: M \rightarrow$ $M$ is a nonzero generalized derivation with associated derivation $d$. If $D$ acts as a homomorphism on $I$ and if $d \neq 0$ on $I$, then $M$ is commutative.

\section{Proof}

If $D$ acts as a homomorphism on $I$, then we have

$$
D(x \alpha y)=D(x) \alpha y+x \alpha d(y)=D(x) \alpha D(y) \text { for all } x, y \in I, \alpha \in \Gamma \text {. }
$$

For any $x, y, z \in I$, we find that

$$
D(x \alpha y \beta z)=D(x \alpha y) \beta z+x \alpha y \beta d(z)=D(x) \alpha D(y) \beta z+x \alpha y \beta d(z) \text { for all } x, y, z \in I, \alpha, \beta \in \Gamma
$$

On the other hand,

$$
D(x \alpha y \beta z)=D(x) \alpha D(y \beta z)=D(x) \alpha D(y) \beta z+D(x) \alpha y \beta d(z) \text { for all } x, y, z \in I, \alpha, \beta \in \Gamma \text {. }
$$

On comparing (2) and (3), we get $(D(x)-x) \alpha y \beta d(z)=0$ for all $x, y, z \in I, \alpha, \beta \in \Gamma$. Thus, primeness of $M$ forces that either $(D(x)-x)=0$ or $d(z)=0$. If $d(z)=0$ for all $z \in I$, then $d=$ 0 , a contradiction. On the other hand if $D(x)=x$ for all $x \in I$, then

$x \alpha y=D(x \alpha y)=D(x) \alpha y+x \alpha d(y)$ for all $x, y \in I, \alpha \in \Gamma$

and hence we find that $x \alpha d(y)=0$.

Replace $x$ by $x \beta z$ in $x \alpha d(y)=0$,

we get $x \beta \operatorname{zod}(y)=0$, for all $x, y, z \in I, \alpha, \beta \in \Gamma$,

Similarly, replacing $x$ by $z \beta x$, we get

$z \beta x \alpha d(y)=0$, all $x, y, z \in I, \alpha, \beta \in \Gamma$,

Subtracting (5) from (4), we get, $[x, z]_{\beta} \alpha d(y)=0$, all $x, y, z \in I, \alpha, \beta \in \Gamma$.

Replacing $y$ by $y \delta r, r \in I$, we get for all $x, y, z, r \in I, \alpha, \beta, \delta \in \Gamma$,

$[x, z]_{\beta} \alpha d(y \delta r)=[x, z]_{\beta} \alpha d(y) \delta r+[x, z]_{\beta} \alpha y \delta d(r)=[x, z]_{\beta} \alpha y \delta d(r)=0$,

Since $d(r) \neq 0$ on $I$, we get $[x, z]_{\beta}=0$, for all $x, z \in I, \beta \in \Gamma$ by the primeness of $M$. By lemma 2.3, $M$ is commutative.

Theorem 2.5. Let $M$ be a prime $\Gamma$-ring satisfying the condition (*) and $I$ be a nonzero ideal of $M$. Suppose $D: M \rightarrow M$ is a nonzero generalized derivation with associated derivation $d$. If $D$ acts as an anti-homomorphism on $I$ and if $d \neq 0$ on $I$, then $M$ is commutative.

\section{Proof}

If $D$ acts as an anti-homomorphism

$$
D(x \alpha y)=D(x) \alpha y+x \alpha d(y)=D(y) \alpha D(x) \text { for all } x, y \in I, \alpha \in \Gamma \text {. }
$$


Replacing $x$ by $x \beta y$ in (6) and (*), we get

$x \alpha y \beta d(y)=D(y) \alpha x \beta d(y)$, for all $x, y \in I, \alpha, \beta \in \Gamma$.

Now, replace $x$ by $z \delta x$ in (7), to get

$z \delta x \alpha y \beta d(y)=D(y) \alpha z \delta x \beta d(y)$, for all $x, y, z \in I, \alpha, \beta, \delta \in \Gamma$

Left multiplying (7) by $z$, we obtain

$z \delta x \alpha y \beta d(y)=z \delta D(y) \alpha x \beta d(y)$, for all $x, y, z \in I, \alpha, \beta, \delta \in \Gamma$

Comparing (8) and (9), we find that $[D(y), z]_{\alpha} \alpha x \beta d(y)=0$, for all $x, y, z \in I, \alpha, \beta \in \Gamma$. Replacing $x$ by $x \lambda r$, we get $[D(y), z]_{\alpha} \delta x \lambda r \beta d(y)=0, x, y, z \in I, r \in M, \alpha, \beta, \delta, \lambda \in \Gamma$. By the primeness of $M$ either $[D(y), z]_{\alpha} \delta x=0$ or $d(y)=0$. By lemma 2.1,either $[D(y), z]_{\alpha}=0$ or $d(y)=0$ Now, let $A=\left\{y \in I \mid[D(y), z]_{\alpha}=0\right.$, for all $\left.z \in I\right\}, B=\{y \in I \mid d(y)=0\}$. Thus $A$ and $B$ are additive subgroups of $I$ and $I=A \cup B$. But a group can not be a union of two proper subgroups and hence $I=A$ or $I=B$. If $I=B$ then $d(y)=0$ for all $y \in I$ and hence $d=0$, a contradiction. On the other hand, if $I=A$, then $[D(y), z]_{\alpha}=0$, for all $y, z \in I, \alpha \in \Gamma$. Now, replace $y$ by $y \lambda z$ to get $[y, z]_{\alpha} \lambda d(z)+y \lambda[d(z), z]_{\alpha}=0$. Again replacing $y$ by $x \delta y$ we get $[x, z]_{\alpha} \delta y \lambda d(z)=0$ for all $x, y, z \in I, \alpha, \delta, \lambda \in \Gamma$. Thus primeness of $M$ implies that for each $z \in I$ either $[x, z]_{\alpha}=0$ or $d(z)=0$. If $d(z)=0$ for all $z \in I$ then $d=0$. Now, if $[x, z]_{\alpha}=0$ for all $x, z \in I, \alpha \in \Gamma$, then by Lemma 2.3 we get the required result. This completes the proof of the theorem.

\section{References}

1. H. E. Bell and L.C. Kappe, Acta Math. Hung. 53, 339 (1989). http://dx.doi.org/10.1007/BF01953371

2. M.S. Yenigul and N. Argac, Turkish J. Math. 18, 280 (1994).

3. M. Ashraf, N. Rehman and M.A. Quadri, Rad. Math. 9, 187 (1999).

4. Nadem-Ur-Rehman, Glasnik Matematicki 39 (59), 27 (2004).

5. A. Ali and D. Kumar, Internat. Math. Forum 2 (23), 1105 (2007).

6. W. E. Barnes, Pacific J. Math. 18, 411 (1966).

7. H. E. Bell and W.S. Martindale, Canad. Math. Bull. 30, 91 (1987).

8. B. Havala, Comm. Algebra 26, 1147 (1998). http://dx.doi.org/10.1080/00927879808826190

9. J. H. Mayne, Canad. Math. Bull. 27, 122 (1984). http://dx.doi.org/10.4153/CMB-1984-018-2

10. N. Rehman, Math. J. Okayama Univ. 44, 43 (2002).

11. M. J. Atteya, Int. Math. J. Algebra 4, 591 (2010). 